\title{
Fulminant hepatic failure caused by tuberculosis
}

\author{
W Hussain, D Mutimer, R Harrison, S Hubscher, J Neuberger
}

\begin{abstract}
A 54 year old Asian woman developed fulminant hepatic failure followed by renal failure. Because of a past history of possible tuberculosis, she was given antituberculous drugs. The chest $x$ ray was normal. A transjugular liver biopsy showed caseating necrosis, granulomas, and acid fast bacilli indicative of miliary tuberculosis. Despite full supportive therapy, her condition deteriorated and she died. Postmortem examination showed widespread miliary tuberculosis; culture confirmed the presence of Mycobacterium tuberculosis. Tuberculosis causes fulminant hepatic failure rarely and only three cases have been described. In this, as with the other cases, hyponatraemia and hepatomegaly were features at presentation. This is the first report of treatment being given before death.

(Gut 1995; 36: 792-794)
\end{abstract}

Keywords: fulminant hepatic failure, tuberculosis.

Most cases of fulminant hepatic failure result from viral infection or drug toxicity. ${ }^{1}$ The treatment of such patients is largely supportive, with liver transplantation being indicated for those with a poor prognosis. It is important, however, to make an early diagnosis of the cause of the fulminant hepatic failure because treatment may be affected. For instance, the prognosis and the use of prognostic markers are dependant on the aetiology; early diagnosis can allow for a decision on the need for transplantation to be made before the onset of irreversible complications. ${ }^{2}$ In some cases the correct diagnosis will lead to alternative intervention, as in the case of fulminant hepatic failure associated with pregnancy, or may contraindicate liver transplantation as in fulminant hepatic failure resulting from malignant infiltration of the liver.

Bacterial infection is a rare cause of fulminant hepatic failure and usually results from Gram negative septicaemia, often in association with pregnancy. Treatment with appropriate antibiotics is usually followed by prompt recovery. We report a woman with disseminated tuberculosis who presented with fulminant hepatic failure and despite early treatment died.

Pathology

R Harrison

$S$ Hubscher

Queen Elizabeth Hospital, Birmingham

Correspondence to: Dr J Neuberger, Liver Unit, Queen Elizabeth Hospital, Birmingham B15 2TH.

Accepted for publication 15 July 1994 A 54 year old Kenyan Asian woman, who had lived in the United Kingdom since 1968, presented on the 10 November 1993 to her local hospital with a five day history of vomiting and right upper quadrant abdominal pain. She had been admitted two months earlier for investigation of chest pain, for which no cause had been found. Liver tests at that time were normal.

Between 1971 and 1973 she had been treated for presumed pulmonary tuberculosis on the basis of abnormal chest $x$ ray and a raised erythrocyte sedimentation rate; sputum culture was repeatedly negative. Further clinical details are not available. Treatment was rifampicin $450 \mathrm{mg}$ once daily, isoniazid $100 \mathrm{mg}$ three times daily, and pyridoxine $10 \mathrm{mg}$ daily.

There was a history of mild asthma for many years for which she took salbutamol inhaler as required, and hypertension treated with amlodipine $5 \mathrm{mg}$ once daily for more than a year. She was not taking any other drugs. There was no history of alcohol consumption or use of recreational drugs. Her sister had been diagnosed as suffering from tuberculosis.

On examination she was mildly jaundiced, with a fever of $39^{\circ} \mathrm{C}$. She was tender in the right upper quadrant of the abdomen, there were no cutaneous stigmata of chronic liver disease. The chest was normal on clinical examination. Investigations showed a raised bilirubin of $66 \mu \mathrm{mol} / 1$ (NR 1-26), alkaline phosphatase 695 U/l (NR 70-380), aspartate transaminase $151 \mathrm{U} / 1$ (NR 5-30), albumin of $24 \mathrm{~g} / \mathrm{dl}$ (NR 34-51), and a serum sodium of $126 \mathrm{mmol} / \mathrm{l}$ (NR 134-146). The prothrombin time was prolonged with an INR of 1.6 (NR $0 \cdot 8-1 \cdot 2)$. Paracetamol was undetectable and subsequent investigations showed that tests for hepatitis A IgM, hepatitis B surface antigen, anti-hepatitis B core (IgM and IgG), and hepatitis C RNA were all negative. The chest $x$ ray was normal, abdominal ultrasound showed a slightly enlarged liver but no other abnormality.

The clinical and biochemical picture deteriorated over the next eight days. The serum aspartate transaminase reached 1790 $\mathrm{U} / \mathrm{l}$ and serum sodium fell to $114 \mathrm{mmol} / \mathrm{l}$, so she was transferred to the Queen Elizabeth Hospital on 19 November 1993 for consideration for possible transplantation. On arrival she was in grade I hepatic encephalopathy but her condition deteriorated over the next 12 hours to grade IV. Mechanical ventilation and inotropes were required. Renal failure developed - the serum creatinine rose to $341 \mu \mathrm{mol} / \mathrm{l}$ (NR 50-101) and the urea to

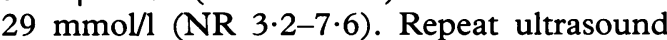
scan showed a homogenously enlarged liver. The chest $x$ ray was again normal.

Because of the possibility that the liver failure was caused by tuberculosis, treatment with rifampicin $600 \mathrm{mg}$, isoniazid $300 \mathrm{mg}$, pyrazinamide $2 \mathrm{~g}$, and pyridoxine $10 \mathrm{mg}$ daily was started; a decision to list her for 


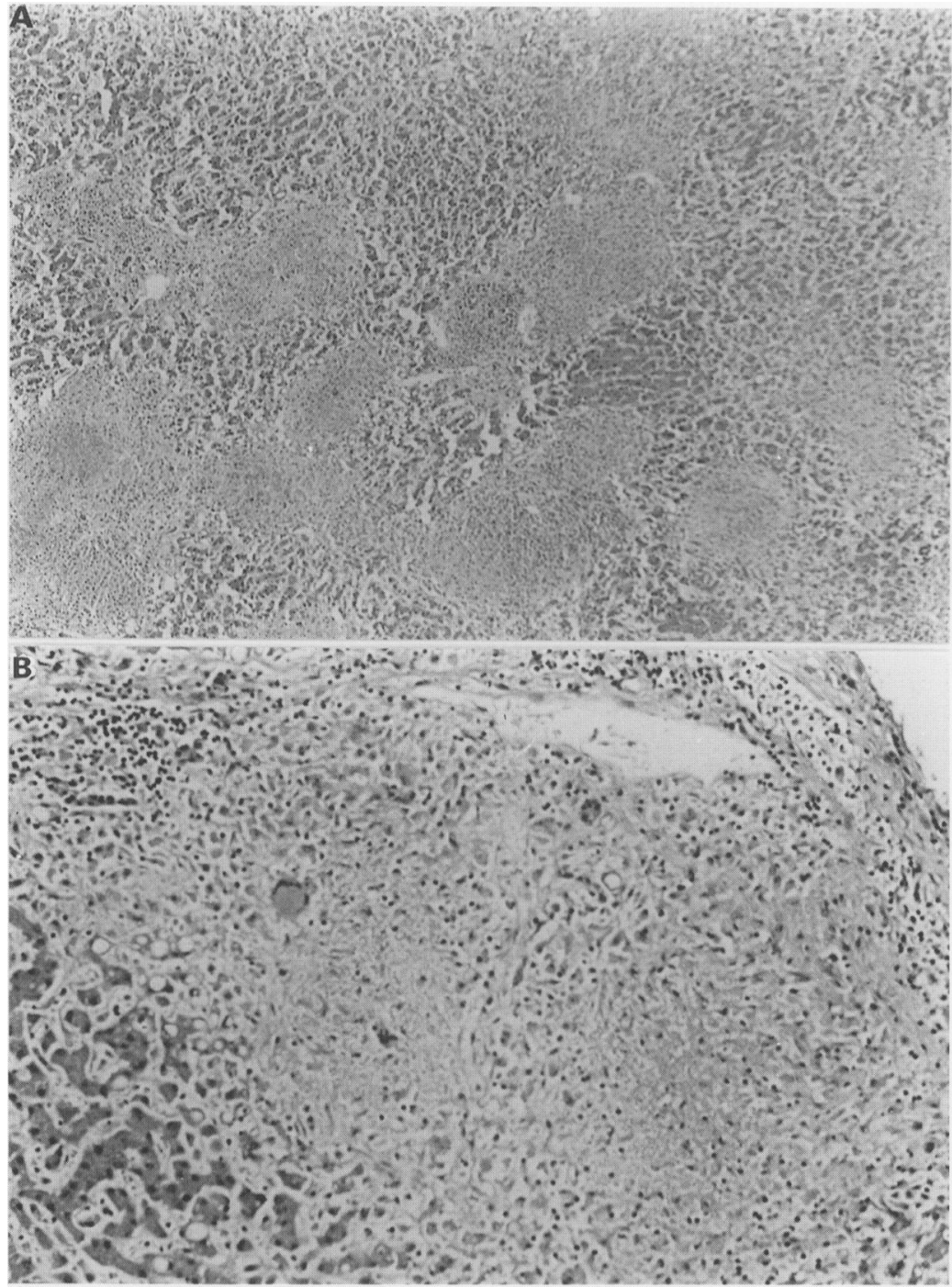

(A) Liver at postmortem examination containing numerous irregular areas of caseation necrosis. Areas of surviving liver parenchyma appear unremarkable (haematoxylin and eosin); (B) higher power view of liver at postmortem examination showing an area of caseation necrosis with a poorly formed granulomatous reaction at the periphery. A solitary giant cell is present.

\section{Discussion}

Although fulminant hepatic failure may occur as part of a systemic illness resulting from tuberculosis, tuberculosis presenting as fulminant hepatic failure has been rarely reported. ${ }^{3-5}$

We believe that this patient's liver failure was caused by tuberculosis for a number of reasons. Viral causes have, as far as possible, been excluded serologically although it is by definition impossible to exclude the putative non-A non-B non- $C$ virus. Furthermore, the histological features, both at transjugular biopsy and at necropsy, did not show features characteristic of fulminant viral hepatitis. ${ }^{6} \mathrm{~A}$ drug related aetiology is unlikely because treatment had been unchanged for over a year. ${ }^{7}$ Postmortem examination failed to show other possible causes of fulminant hepatic failure such as malignant infiltration, ischaemia or extrahepatic biliary obstruction. The liver biopsy and postmortem examination both showed features of hepatic necrosis, granulomatous infiltration, and on staining acid fast bacilli. The degree of hepatocyte necrosis is sufficient to cause the clinical picture of liver failure.

It is well established that miliary tuberculosis can affect the liver. One series reported hepatic granulomata in all cases of miliary tuberculosis studied. ${ }^{8}$ Although microscopic involvement of the liver is common, clinical consequences are rare. Cruise ${ }^{9}$ reported clinical jaundice in only seven cases of 1748 patients hospitalised for tuberculosis. Hyponatraemia is not uncommon, however, in patients with disseminated tuberculosis ${ }^{10}$; this was not a prominent feature of this case. Cases of tuberculosis progressing to fulminant hepatic failure have been reported. Godwin ${ }^{3}$ reported a case of a 67 year old man who presented with myalgia and upper respiratory symptoms then developed fulminant hepatic and renal failure who on postmortem examination was found to have miliary tuberculosis. However, other potential causes of fulminant hepatic failure, such as hepatitis C or drugs, were not excluded.

There are also two case reports from Japan of patients similar to the one reported here. ${ }^{45}$ The time from admission to death was also very rapid - three and 11 days. In these cases, as with our own, the liver was enlarged. Fulminant hepatic failure, resulting from viruses, drugs or toxins is usually associated with a shrunken liver. The enlarged liver in this and the other cases of miliary tuberculosis, together with hyponatraemia, may be a useful pointer to this unusual cause of fulminant hepatic failure. The use of a transjugular liver biopsy permitted early diagnosis. This resulted in what we believe is the first case where the diagnosis was made and treatment started before death. The clinical and serological features suggested that the prognosis was extremely poor and transplantation was, therefore, considered. Because of the presence of active untreated tuberculosis, however, it was felt that transplantation would be unsuccessful as the effect of surgery and immunosuppression would make it highly probable that she would die from disseminated tuberculosis. The There was no significant inflammation and no other features were present to suggest recent viral hepatitis of any other cause for liver failure.

Culture of the liver specimen confirmed the presence of Mycobacterium tuberculosis. 
fact that viable mycobacteria were found on postmortem examination even two days after the start of appropriate antibiotic treatment, suggests that there was active disease, which would have become increasingly active after transplantation.

Fulminant hepatic failure caused by tuberculosis is rare in this country, but the incidence of tuberculosis infection is increasing. Although this patient died despite treatment, this rare cause of fulminant hepatic failure should be considered in patients at risk so treatment can be started early.

1 Lee W. Acute liver failure. $N$ Engl f Med 1993; 329: $1862-72$.

2 O'Grady J, Alexander GJM, Hayllar KM, Williams R. Early indicators of prognosis in fulminant hepatic failure. Gastroenterology 1989; 97: 439-45.
3 Godwin JE, Coleman AA, Sahn SA. Miliary tuberculosis presenting as hepatic and renal failure. Chest 1991; 99: $752-4$.

4 Asada Y, Hayashi T, Sumiyoshi A, Aburaya M, Shishime E. Miliary tuberculosis presenting as fever and jaundice with hepatic failure. Hum Pathol 1991; 22: 92-4.

5 Hosokawa K, Morita A, Imai T, Kitahara M, Kamegaya K, Tanaka $M$, et al. A case of miliary tuberculosis presenting as fever and jaundice with hepatic failure, looking like the course of fulminant hepatitis. Kansenshogaku Zasshi 1992; 66: 1288-92.

6 Scheuer PJ. Acute hepatitis. In: Wight DGD, ed. Systemic pathology. 3rd ed. Vol II. Liver, biliary tract and pancreas. Edinburgh: Churchill Livingstone, 1994 53-70.

7 Danan G. Concensus meeting. Criteria of drug induced liver disorders. f Hepatol 1990; 11: 272-6.

8 Cucin RL, Coleman M, Eckhardt JJ, Silvet RT. The diagnosis of miliary tuberculosis: utility of peripheral blood abnormalities, bone marrow and liver biopsy. fournal of Chronic Diseases 1974; 26: 355-61.

9 Cruise JM. Jaundice in tuberculosis. Am F Med Sci 1974; 147: 720-6.

10 Munt RW. Miliary tuberculosis in the chemotherapy era: with a clinical review in 69 American adults. Medicine 1972; 51: 139-55. 